\title{
Bioassay-Guided Isolation and HPLC Determination of Bioactive Compound That Relate to the Antiplatelet Activity (Adhesion, Secretion, and Aggregation) from Solanum lycopersicum
}

\author{
Eduardo Fuentes, ${ }^{1,2,3}$ Ricardo Castro, ${ }^{3}$ Luis Astudillo, ${ }^{2,3}$ Gilda Carrasco, ${ }^{2,4}$ \\ Marcelo Alarcón, ${ }^{1,2}$ Margarita Gutiérrez, ${ }^{2,3}$ and Iván Palomo ${ }^{1,2}$ \\ ${ }^{1}$ Immunology and Haematology Laboratory, Immunohaematology and Clinical Biochemistry Department, Faculty of Health Sciences, \\ Universidad de Talca, 3460000 Talca, Chile \\ ${ }^{2}$ Centro de Estudios en Alimentos Procesados (CEAP), CONICYT-Regional, Gore Maule, R09I2001 Talca, Chile \\ ${ }^{3}$ Synthesis Laboratory, Chemical Institute of Natural Resources, Universidad de Talca, 3460000 Talca, Chile \\ ${ }^{4}$ Horticulture Department, Faculty of Agricultural Sciences, Universidad de Talca, 3460000 Talca, Chile
}

Correspondence should be addressed to Margarita Gutiérrez, mgutierrez@utalca.cl and Iván Palomo, ipalomo@utalca.cl

Received 5 September 2012; Accepted 8 October 2012

Academic Editor: Adair Roberto Soares Santos

Copyright ( 92012 Eduardo Fuentes et al. This is an open access article distributed under the Creative Commons Attribution License, which permits unrestricted use, distribution, and reproduction in any medium, provided the original work is properly cited.

In seeking the functionality of foodstuff applicable to medicine, ripe tomato fruits were found to show an antiplatelet activity. Therefore, the bioactive compound was isolated, structurally identified, and studied for an inhibitory effects on platelet adhesion, secretion, and aggregation. The concentration of adenosine in ripe tomato fruits (pulp and skin extracts) and its processing byproducts (paste and pomace) was determined by reversed-phase high-performance liquid chromatography (HPLC). According to platelet aggregation inhibition induced by ADP, the total extract residual was fractionated by liquid-liquid separation, obtaining aqueous, ethyl acetate and petroleum ether extracts. The aqueous extract was subjected to repeated permeation over sephadex LH20 and semipreparative TLC. The isolate finally obtained was identified as adenosine on the basis of ESI-MS, ${ }^{1} \mathrm{H}$ NMR, HPLC, and UV spectra. Adenosine concentration dependently $(2.3-457 \mu \mathrm{M})$ platelet aggregation inhibited induced by ADP. Also, adenosine present inhibition of platelet secretion and thrombus formation under flow conditions. The quantitative HPLC analysis revealed significant amounts of adenosine in ripe tomato fruits and its processing by-products. From these results, extracts/fractions of ripe tomato fruits and their processing by-products may be referred to as functional food and functional ingredients containing a compound that inhibits platelet function with a potent preventive effect on thrombus formation, as those that occur in stroke.

\section{Introduction}

According to the World Health Organization, cardiovascular disease (CVD) (i.e., acute myocardial infarction, cerebrovascular disease, and peripheral arterial thrombosis) represents about $30 \%$ of deaths worldwide [1], with a relative increase over time due to the aging of the population [2].

The current lifestyle of the population contributes to the development of risk factors for CVD, such as hypertension, diabetes, smoking, and hypercholesterolemia [3, 4]. The development and progression of CVD lie in the interactive processes of atherosclerotic lesions and thrombus formation, an interaction established primarily by platelet participation [5].

In the context of atherosclerosis, platelets can adhere to endothelial cells and contribute to the recruitment of leukocytes involved in the local vascular inflammation $[6,7]$. Platelets also intensify the inflammatory process at all stages of atherosclerosis by expressing membrane molecules such as intercellular adhesion molecule-2, P-selectin, and CD40L $[8,9]$. Also, when there is a damaged atheromatous plaque, platelets adhere, secrete their contents, and then aggregate 
to it [10]. This activation is a dynamic process that can lead to intermittent or permanent obstruction to blood flow, resulting in ischemic tissue injury and organ dysfunction [11].

From the point of view of public health, efforts should be directed to primary prevention, namely, to reduce cardiovascular risk factors mentioned above $[12,13]$. In this context, regular consumption of fruits and vegetables, apart of the called Mediterranean diet [14], might be related to the bioactive compounds found in them [15], which explains the increasing amount of attention in research on phytochemicals in the prevention of CVD [16]. Tomatoes (Solanum lycopersicum), fresh or processed (e.g., tomato paste), apart from their nutritional value, have been found to provide a cardioprotective effect, both at the endothelial and platelet levels [17].

The purpose of this paper was to isolate and identify one of the bioactive compounds of tomatoes that present antiplatelet activity (adhesion, secretion, and aggregation) and also to examine extensively the effect of different kinds of tomato processing, in the production of paste and its byproduct pomace on the content of antiplatelet compound.

\section{Materials and Methods}

2.1. Chemicals and Reagents. Acetonitrile HPLC grade from Merck (Darmstadt, Germany) was used as well as Sephadex LH-20 (Pharmacia Fine Chemicals, Piscataway, NJ, USA) and thin layer chromatography (TLC) plates (Merck, Darmstadt, Germany), whereas acetylsalicylic acid, 1,1-diphenyl2-pycrylhydrazyl (DPPH), quercetin, catechin, and adenosine were purchased in Sigma-Aldrich (St. Louis, Missouri/MO, USA). Sodium chloride (p.a.), potassium chloride (p.a.), sodium phosphate dibasic (p.a.), potassium phosphate monobasic (p.a.), petroleum ether, methanol, and ethyl acetate were obtained from Arquimed (Santiago, Chile). The agonist adenosine $5^{\prime}$-diphosphatebis (ADP), calcein-AM, collagen, and bovine serum albumin (BSA) were obtained in Sigma-Aldrich (St. Louis, Missouri/MO, U.S.A), whereas luciferase luciferin reagent was obtained from Chrono-Log corp. (Havertown, PA) and microfluidic chambers were from Bioflux (Fluxion, San Francisco, California, USA).

2.2. Processing Material. Processing tomato (H9665, H9997, and H7709), pomace and tomato paste were obtained from "Tresmontes Luchetti" (Production plant Talca, Chile).

2.3. Industrial Quality Measurement. The industrial quality through firmness expressed in pounds (McCormick Fruit Pressure Tester model FT 327, Yakima, Wash) and soluble solids expressed in ${ }^{\circ}$ Brix (Meiji-La Bax HT 0-32) were determined from a sample of harvested fruits (10/experimental unit).

2.4. Approximate Chemical Composition. To characterize the chemical composition of the pulp of tomatoes, the water content (\%) was determined by drying in a convection oven at $60^{\circ} \mathrm{C}$, the protein content was measured using the Kjeldahl method, and fat content was determined by Soxhlet method. Ash content was obtained by drying the samples at $550^{\circ}$ in a muffle furnace $\mathrm{C}$ for two hours. Crude fiber was determined by the acid sequence method using $1.25 \% \mathrm{H}_{2} \mathrm{SO}_{4}$ and $1.25 \% \mathrm{NaOH}$ for acid and alkaline hydrolysis, respectively. Carbohydrate content was calculated as the difference between the total and the contents of all other ingredients [18]. Each measurement was performed in triplicate.

2.5. Extraction and Fractionation. Tomatoes were carefully washed; skin and seeds were manually separated from the pulp. Then the pulp was homogenized in a blender (Somela BL1500) and extracted three times with $\mathrm{MeOH}$ $(3 \times 15 \mathrm{~L}$ each $)$ at room temperature in the dark for $12 \mathrm{~h}$ per extraction. The mixture was sonicated (Elma Transsonic 700/H, Singen, Germany) for $5 \mathrm{~min}$ and then filtered through gauze twice. The filtrate was evaporated under vacuo (Laborata 4001, Heidolph, Germany or RE 111-B461, Buchi Labortechnik, The Netherlands) to remove methanol. The total extract residuary was fractionated by liquid-liquid separation, obtaining petroleum ether, ethyl acetate, and aqueous extracts. Since the aqueous extract had significant antiplatelet properties further purification was carried out. The aqueous extract was lyophilized (Freezone 6, Kansas City, Missouri, Labconco, USA).

2.5.1. Isolation. The aqueous extract was subjected to a repeated permeation over Sephadex LH-20 (column length $60 \mathrm{~cm}$, internal diameter $3 \mathrm{~cm}$ ) using $\mathrm{MeOH}: \mathrm{H}_{2} \mathrm{O} 4: 1$ as eluent. The fractions were monitored at $254 \mathrm{~nm}$ using a He入ios R V-3.06 spectrophotometer (Unicam spectrometry, Cambridge, UK) and analyzed by HPLC $\left(\mathrm{A}: \mathrm{H}_{2} \mathrm{O}\right.$ and $\mathrm{B}$ : acetonitrile; $0-10$ min linear gradient $98-2 \% \mathrm{~B}$, and 10 20 min linear gradient $95-5 \%$ B, 20-30 min linear gradient $50-50 \% \mathrm{~B}$, and finally, washing the column with $100 \% \mathrm{~B}$ for $10 \mathrm{~min})$.

Semipreparative TLC was performed on $10 \mathrm{~cm} \times 20 \mathrm{~cm}$ TLC silica gel plates coated with $1 \mathrm{~mm}$ layer and the sample was applied. The plate was developed using $25 \mathrm{~mL}$ of mobile phase EtOAc:AcOH: $\mathrm{H}_{2} \mathrm{O} \quad 10: 2: 3 \mathrm{v} / \mathrm{v} / \mathrm{v}$ in a saturated chamber. The plate under UV light $(254 \mathrm{~nm})$ was developed. The bands observed were removed, extracted with methanol, and concentrated. Since one of the bands had significant antiplatelet properties, further identification was carried out.

\subsection{Identification of Antiplatelet Compound}

2.6.1. Spectral Scanning. Spectral scanning between 200 and $600 \mathrm{~nm}$ was used to investigate the UV-visible maximum band absorption. The UV/Vis spectra were obtained in a spectrophotometer using $\mathrm{MeOH}$ as a solvent.

2.6.2. NMR Analysis. The structure of band was determined by ${ }^{1} \mathrm{H}$ NMR on a Bruker AMX spectrometer (Bruker, Germany) operating at $400 \mathrm{MHz}$, using $\mathrm{DMSO}^{-\mathrm{D}_{6}}$ as a solvent. TMS was used as an internal standard. Chemical shifts $(\delta)$ 
and $J$ values were reported in $\mathrm{ppm}$ and $\mathrm{Hz}$, respectively, relative to the solvent peak $\left(\mathrm{DMSO}-\mathrm{D}_{6}\right.$ at 2.50 and $3.34 \mathrm{ppm}$ for protons). Signals were designated as follows: s, singlet; d, doublet; $\mathrm{dd}$, doublet of doublets; $\mathrm{t}$, triplet; $\mathrm{m}$, multiplet.

2.6.3. Mass Spectrometer. ESI-MS/MS data was collected using a high resolution hybrid quadrupole $(\mathrm{Q})$ and orthogonal time-of-flight (TOF) mass spectrometer (Micromass QTof, UK) with constant nebulizer temperature of $80^{\circ} \mathrm{C}$. The ESI source and the mass spectrometer were operated in a negative ion mode, and the cone and extractor potentials ware of $10 \mathrm{eV}$, with a scan range of $m / z \quad 100-500$. The band infused into the ESI source at flow rates of $5 \mu \mathrm{L} \mathrm{min}{ }^{-1}$ was dissolved in acetonitrile ion-induced dissociation (CID) with argon in the collision chamber. The values expressed are average mass and correspond to the $[\mathrm{M}-\mathrm{H}]^{-}$ion.

2.7. Preparation of the Standard Curve and Extracts for Quantitative Analysis. Standard solutions of adenosine $3.75 \mathrm{mg} / \mathrm{mL}$ for quantification were prepared dissolving $187.5 \mathrm{mg}$ in a volumetric flask of $50 \mathrm{~mL}$ (PBS: phosphate buffered saline $\mathrm{pH} 7.4,0.1 \mathrm{M}$ ). The points of the curve were realized by the dilution of the stock solution between 3.75 and $0.19 \mathrm{mg} / \mathrm{mL}$ to produce a sequence of $3.75,1.88$, 0.75 and $0.19 \mathrm{mg} / \mathrm{mL}$ in buffer PBS. Extracts of ripe tomato fruits (pulp and skins extracts) and their by-products of processing (paste and pomace extracts) were lyophilized [19] and then equilibrated to room temperature for $1 \mathrm{~h}$ and dissolved in $1000 \mu \mathrm{L}$ of PBS. The content of adenosine was expressed in $\mathrm{mg}$ adenosine/mg dried extract. All samples were filtered through a Millex-LS PTFE filter with $5 \mu \mathrm{m}$ pore size (Millipore Corporation, Billerica, MA, USA); before HPLC analysis, the injection was made in duplicate for $100 \mu \mathrm{L}$.

2.8. HPLC. The quantitative study of the adenosine in ripe tomato fruits and its by-products of processing extracts were performed by HPLC. The HPLC system (Agilent ChemStation, 1200, USA) consisted of a low-pressure quaternary pump (model Agilent 1200) and autosampler (model Agilent 1260 Infinity Autosampler) with 99-vial capacity sample. Separations were carried out on a LiChrospher RP18 column of $250 \mathrm{~mm}(5 \mu \mathrm{m})$ particle size. A guard column (LiChrospherRP) select B $(5 \mu \mathrm{m})$ particle size was placed in front of the analytical column. The chromatographic conditions were the following: PBS mobile phases which were subjected to filters $(5.0 \mu \mathrm{m})$ and gradient programmed isocratic, room temperature, run time 23 minutes, injection volumes $100 \mu \mathrm{L}$, and wavelengths 254 [20].

\subsection{Antioxidant Activity}

2.9.1. DPPH Free Radical Scavenging Assay. The scavenging activity of the extracts was estimated using DPPH as the free radical model according to the method adapted from Molyneux [21]. An aliquot of $750 \mu \mathrm{L}$ of samples and control ( $80 \%$ methanol) were mixed, respectively, with $1.5 \mu \mathrm{L}$ of DPPH for final concentration of 100,500 , and $1000 \mu \mathrm{g} / \mathrm{mL}$.
The mixture was shaken vigorously and left to stand at room temperature for $30 \mathrm{~min}$ in the dark. The mixture was measured spectrophotometrically at $515 \mathrm{~nm}$. The free radical scavenging activity was calculated as percentage of DPPH discoloration using the following equation (1): percentage of scavenging DPPH free radical $=100 \times(1-$ $\mathrm{AE} / \mathrm{AD}$ ), where $\mathrm{AE}$ is the absorbance of the solution after adding the extract at a particular level, and $\mathrm{AD}$ is the absorbance of the blank DPPH solution. Quercetin and catechin were used as reference compounds. Each measurement was performed in triplicate.

\subsection{Effect on Platelet Function}

2.10.1. Preparation of Human Platelet Suspensions. Venous blood samples were taken from two volunteers (healthy university students), who previously signed informed consent in $3.2 \%$ citrate tubes $(9: 1 \mathrm{v} / \mathrm{v})$ by phlebotomy with vacuum tube system (Becton Dickinson Vacutainer Systems, Franklin Lakes, NJ, USA). The protocol was authorized by the Ethic Committee of Universidad de Talca in accordance with the Declaration of Helsinki (approved by the 18th World Medical Assembly in Helsinki, Finland, 1964). The samples were gently homogenized by 5 -fold inversion and allowed to stand for 5 minutes. Then they were centrifuged (DCS16 Centrifugal Presvac RV) at $240 \mathrm{~g}$ for 10 minutes, and $1 \mathrm{~mL}$ of platelet-rich plasma (PRP) was taken from each tube for platelet count (in triplicate) in an hematologic counter (Bayer Advia 60 Hematology System, Tarrytown, NY, USA). The original tubes were centrifuged at $650 \mathrm{~g}$ for 10 minutes to obtain the platelet-depleted plasma (PDP). Finally, the PRP was adjusted to $2 \times 10^{5}$ platelets $/ \mu \mathrm{L}$ with PDP.

2.10.2. Platelet Antiaggregating Activity. Platelet aggregation was monitored by light transmission turbidimetric method according to Born and Cross [22], using a lumiaggregometer (Chrono-Log, Havertown, PA, USA). Briefly, $480 \mu \mathrm{L}$ of PRP in the reaction vessel were preincubated with $20 \mu \mathrm{L}$ of sample, negative control (saline $0.9 \%$ ), or positive control (acetylsalicylic acid $110 \mu \mathrm{M}$ ). After 3 min of incubation, $20 \mu \mathrm{L}$ of agonist was added to initiate platelet aggregation, which was measured for $6 \mathrm{~min}$. ADP $8 \mu \mathrm{M}$ was used as an agonist. All measurements were performed in triplicate. The results of platelet aggregation (maximum aggregation (\%), slope, area under and lag time (s)) were determined by the software AGGRO/LINK (Chrono-Log, Havertown, PA, USA) and the relative inhibition of the maximum platelet aggregation: $100-((\% \mathrm{AgX} * 100) / \% \mathrm{AgC})(\% \mathrm{AgX}$ : relative aggregation of the component under study, \% AgC: relative control aggregation).

2.10.3. Platelet Secretion Assay. Platelet secretion was determined by measuring the release of ATP using luciferin/ luciferase reagent. Luciferin/luciferase $(50 \mu \mathrm{L})$ was added to $480 \mu \mathrm{L}$ of platelet suspension (PRP adjusted to $2 \times$ $10^{5}$ platelets $/ \mu \mathrm{L}$ ) within 2 min before stimulation. Platelet secretion was recorded in real time in a lumiaggregometer at $37^{\circ} \mathrm{C}$ with stirring $(1000 \mathrm{rpm})$ and luminescence $(\times 0.2)$. 
To examine the effects on platelet secretion, platelets were preincubated with aqueous extract and adenosine for $2 \mathrm{~min}$ prior to the addition of ADP $8 \mu \mathrm{M}[23]$.

\subsubsection{Analysis of Platelet Adhesion and Thrombus Formation} in Flowing Whole Blood. For flow experiments, BioFlux 200 flow system (Fluxion, San Francisco, California, USA) with high shear plates ( 48 wells, $0-20$ dyne $/ \mathrm{cm}^{2}$ ) was used. Using manual mode in the BioFlux software, the microfluidic chambers were coated for 1 hour with $50 \mu \mathrm{L}$ of collagen $200 \mu \mathrm{g} / \mathrm{mL}$ at a wall shear rate of $200 \mathrm{~s}^{-1}$.

The plaque coating was allowed to dry at room temperature for one hour. The channels were perfused with PBS for $10 \mathrm{~min}$ at a wall shear rate of $200 \mathrm{~s}^{-1}$ for removing the interface. Then, the channels were blocked with BSA $0.5 \%$ for $10 \mathrm{~min}$ at a wall shear rate of $200 \mathrm{~s}^{-1}$. In order to visualize platelets, the citrate-anticoagulated blood containing calcein-AM $(4 \mu \mathrm{M})$ was added to the inlet well, and chambers were perfused for $10 \mathrm{~min}$ at a wall shear rate of $1000 \mathrm{~s}^{-1}$.

The plaque-coated microfluidic high shear plates were mounted on the stage of an inverted fluorescence microscope (TE200, NIKON, Japan). Control blood (saline 0.9\%) and blood with aqueous extract $(1 \mathrm{mg} / \mathrm{mL})$ or adenosine $(114 \mu \mathrm{M})$ were preincubated at room temperature for 1 hour prior to the start of flow, and experiments were performed at room temperature [24].

Platelet deposition was observed and recorded in real time (30 frames per min) with a CCD camera (QICAM, QIMaging, Surrey, BC, Canada). We used bright field and fluorescence microscopy for real-time visualization of platelet adhesion and aggregation in flowing blood. For each flow experiment, perfused surface fields of the size of $237900 \mu \mathrm{m}^{2}$ (located in the middle of the channels of the viewing window) were recorded, and fluorescence images were later analyzed offstage by quantifying the area covered by platelets with the Image J software (version $1.26 \mathrm{t}$, NIH, USA). In each field, the areas covered by platelets were quantified.

2.11. Statistical Analysis. Mean \pm standard deviations (SD) were determined using SPSS version 17.0. The data was statistically analyzed by Student's paired or unpaired $t$ test. A Pearson correlation test was used to evaluate the correlation between the adenosine content and inhibition platelet aggregation. The statistical significance level was set up at $P<0.05$.

\section{Results}

3.1. Industrial Quality Measurement. Processing tomatoes presented firmness of $5.2 \pm 0.2$ pounds and soluble solid content of $5.5 \pm 0.8^{\circ}$ Brix, thus a ripening stage VI (red), according to the method proposed by Dumas et al.(2003) [25].

3.2. Characteristics of Tomato Pulp. The approximate chemical composition of tomato pulp showed moisture $94 \pm 2 \%$, protein $12 \pm 0.1 \%$, fat $3 \pm 0.1 \%$, ash $15 \pm 0.1 \%$, carbohydrate
$63 \pm 0.4 \%$, and crude fiber $7 \pm 0.2 \%$, values in concordance with Fuentes et al. [19].

Eighteen grams $(18 \mathrm{~g})(0.3 \% \mathrm{w} / \mathrm{w}$ yield $)$ of a yellow aqueous extract were obtained from $6 \mathrm{~kg}$ of tomato pulp. Such extract showed the highest yield over ethyl acetate extract $(0.05 \% \mathrm{w} / \mathrm{w}$ yield $)$ and petroleum ether extract $(1.4 \times$ $10^{-3} \% \mathrm{w} / \mathrm{w}$ yield).

When comparing different types of extracts, differences in their antioxidant potential were significant. At a concentration of $1000 \mu \mathrm{g} / \mathrm{mL}$, ethyl acetate extract $(87 \pm 2 \%)$ was superior to ether petroleum $(6 \pm 2 \%, P<0.05)$ and aqueous extract $(15 \pm 5 \%, P<0.05)$.

\subsection{Bioassay-Guided Isolation of Antiplatelet Compound.} To advance in the search for a bioactive compound with antiplatelet activity, the aqueous extract was subjected to repeated permeation over Sephadex LH-20 using $\mathrm{MeOH}: \mathrm{H}_{2} \mathrm{O} 4: 1$ as eluent and 21 fractions of $17 \mathrm{~mL}$ each were collected. The fractions were monitored at $254 \mathrm{~nm}$ and two subfractions were identified by HPLC (subfractions $\mathrm{A}$ and $\mathrm{B}$ ). Since platelet aggregation induced by ADP was completely inhibited by subfraction B at $1 \mathrm{mg} / \mathrm{mL}$ further purification was carried out (Figure 1(a)).

The subfraction B ( $50 \mu \mathrm{g}$ by plate) was applied on semipreparative TLC. Under UV light $(254 \mathrm{~nm})$ three bands (BA, BB, and BC) were observed and removed, and extracted with methanol. Then each band was filtered and evaporated under vacuo. Since platelet aggregation induced by ADP was completely inhibited by BC at $1 \mathrm{mg} / \mathrm{mL}$, further identification of compound was carried out.

3.4. Identification of the Antiplatelet Compound. The BC band was identified as adenosine according to the UV spectrum $\left(\lambda_{\max }=221\right.$ and $\left.261 \mathrm{~nm}\right)$; it had an $[\mathrm{M}-\mathrm{H}]^{-}$ at $m / z 266.7856$ [26] and a retention, time similar at adenosine standard $\left(R_{t}=7.8 \mathrm{~min}\right.$ by HPLC) (Figure $1(\mathrm{~b})$ ). The structure was confirmed by NMR spectroscopy. The ${ }^{1} \mathrm{H}$ NMR spectrum of BC was consistent with the structure of adenosine $\delta(\mathrm{ppm}): 8.347(1 \mathrm{H}, \mathrm{s}) ; 8.133(1 \mathrm{H}, \mathrm{s}) ; 7.353(2 \mathrm{H}$, s); $5.870(1 \mathrm{H}, \mathrm{d}, J=6.36 \mathrm{~Hz}) ; 5.44(2 \mathrm{H}, \mathrm{m}) ; 5.15(1 \mathrm{H}, \mathrm{m})$; $4.610(1 \mathrm{H}, \mathrm{m}, J=6.00,11.40 \mathrm{~Hz}) ; 4.136(1 \mathrm{H}, \mathrm{m}) ; 3.960(1 \mathrm{H}$, m); $3.365(1 \mathrm{H}, \mathrm{m}) ; 3.549(1 \mathrm{H}, \mathrm{m})$; for $\mathrm{C}_{10} \mathrm{H}_{13} \mathrm{~N}_{5} \mathrm{O}_{4}$ found following is obtained: $\mathrm{C}: 44.94 \mathrm{H}: 4.90 \mathrm{~N}: 26.21$ and O: 23.95 . The data obtained was consistent with previous reports [27].

3.5. Effect of Aqueous Extract and Adenosine on Platelet Secretion and Aggregation. The results of platelet aggregation induced by the agonist ADP with extract, fraction, and adenosine (band C) are presented in Table 1. After the liquidliquid separation, the inhibition of platelet aggregation induced by $\mathrm{ADP}$ in aqueous extract increased to $54 \pm 8 \%$ $(P<0.05)$. As well as inhibiting the platelet aggregation, the aqueous extract inhibited the platelet secretion in $50 \pm 5 \%$.

The inhibited ADP-induced platelet aggregation of adenosine was concentration dependent $(2.3-457 \mu \mathrm{M})$, in which a concentration of $4.6 \mu \mathrm{M}$ inhibited $50 \pm 12 \%$ platelet aggregation $(P<0.05)$. At the same concentration, it completely inhibited platelet secretion (Figure 2). Besides 
TABLE 1: Effect of extracts, fraction, and antiplatelet compound on platelet aggregation.

\begin{tabular}{|c|c|c|c|c|}
\hline & \multicolumn{4}{|c|}{$\mathrm{ADP}$} \\
\hline & Maximum aggregation (\%) & Slope & Area under the curve & Lag time $(\mathrm{s})$ \\
\hline Total extract $(1 \mathrm{mg} / \mathrm{mL})$ & $51 \pm 8^{*}$ & $38 \pm 2^{*}$ & $206 \pm 10^{*}$ & $24 \pm 3$ \\
\hline Aqueous extract $(1 \mathrm{mg} / \mathrm{mL})$ & $37 \pm 2^{*}$ & $53 \pm 7^{*}$ & $140 \pm 56^{*}$ & $38 \pm 5$ \\
\hline Subfraction B (1 mg/mL) & $1 \pm 8^{*}$ & $6 \pm 6^{*}$ & $2 \pm 3^{*}$ & $>120^{*}$ \\
\hline \multicolumn{5}{|l|}{ Adenosine } \\
\hline $2.3 \mu \mathrm{M}$ & $56 \pm 2^{*}$ & $58 \pm 2^{*}$ & $261 \pm 9^{*}$ & $27 \pm 1$ \\
\hline $4.6 \mu \mathrm{M}$ & $40 \pm 5^{*}$ & $44 \pm 6^{*}$ & $201 \pm 14^{*}$ & $34 \pm 1$ \\
\hline $43 \mu \mathrm{M}$ & $26 \pm 8^{*}$ & $18 \pm 12^{*}$ & $123 \pm 15^{*}$ & $20 \pm 1$ \\
\hline $457 \mu \mathrm{M}$ & $21 \pm 6^{*}$ & $28 \pm 8^{*}$ & $98 \pm 11^{*}$ & $53 \pm 2^{*}$ \\
\hline Negative control & $85 \pm 2$ & $104 \pm 14$ & $393 \pm 21$ & $30 \pm 1$ \\
\hline
\end{tabular}

Values were presented in mean $\pm \mathrm{SD}(n=3)$. Band $\mathrm{C}$ corresponds to adenosine.

ADP $8 \mu \mathrm{M} .{ }^{*} P<0.05$ versus negative control (saline $0.9 \%$ ).
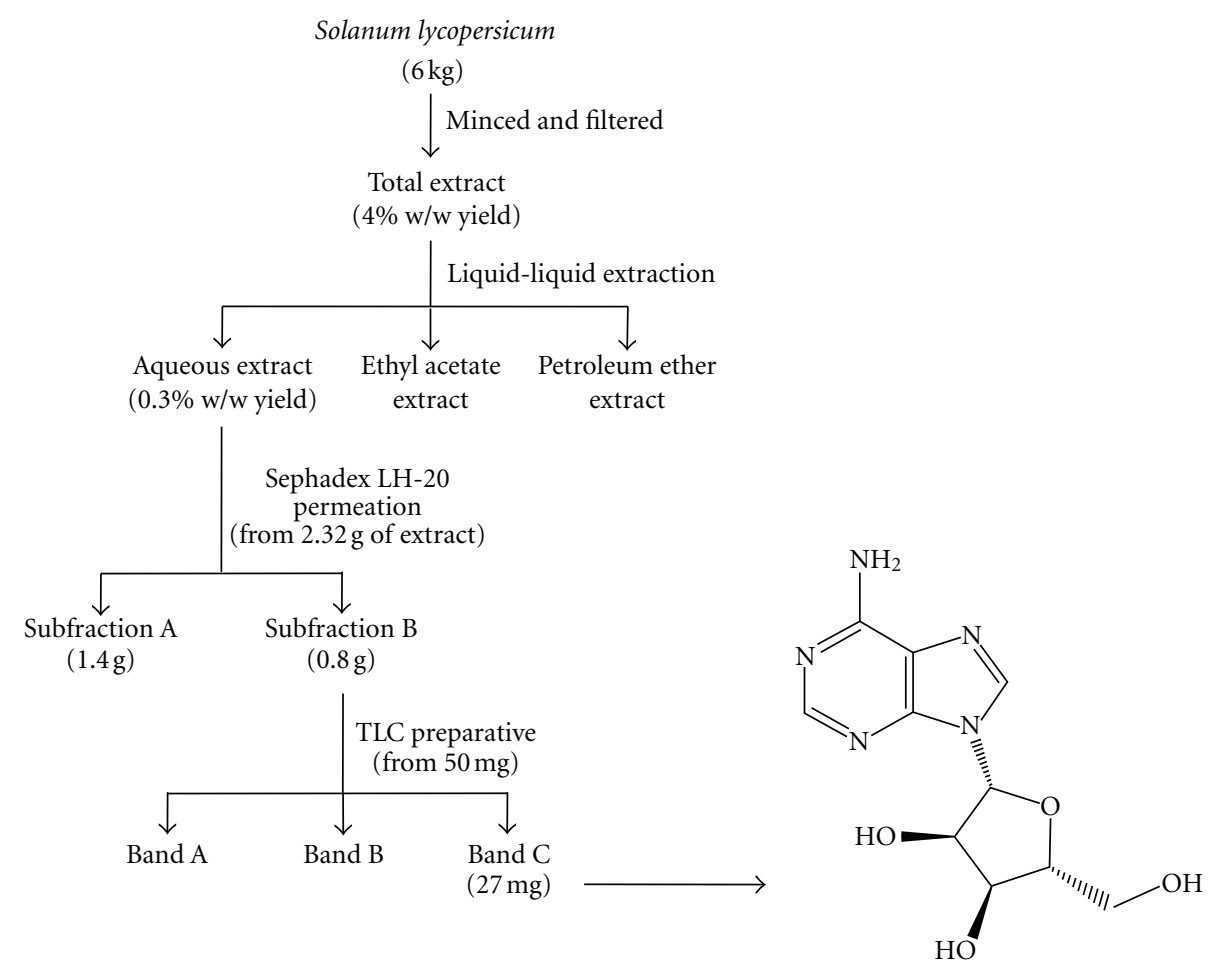

Adenosine

(a)
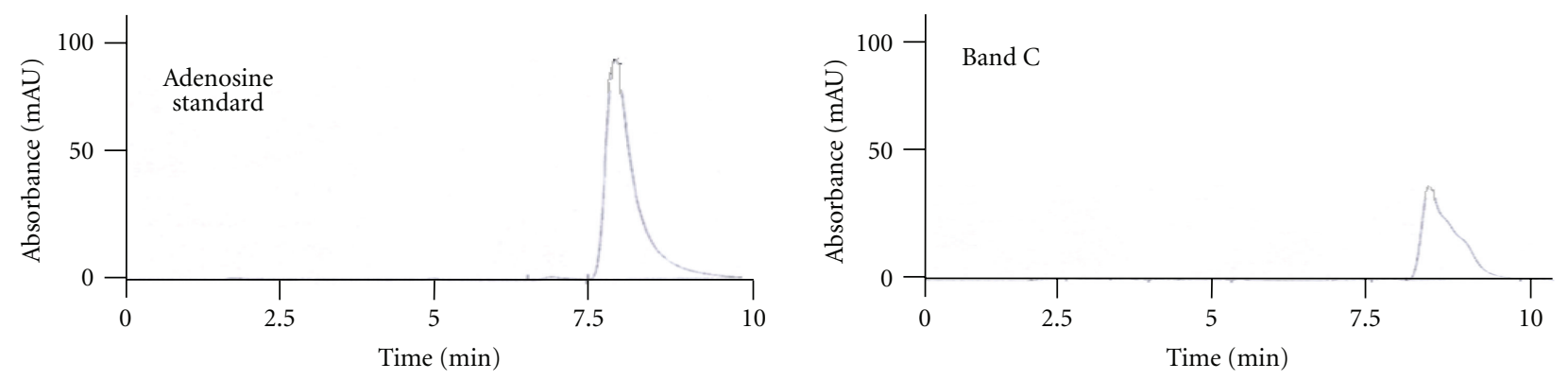

(b)

FIGURE 1: Biodirected isolation and identification of adenosine from S. lycopersicum. (a) Extraction and fractionation of pulp from S. lycopersicum and (b) chromatograms of adenosine standard and Band C dissolved in PBS. 

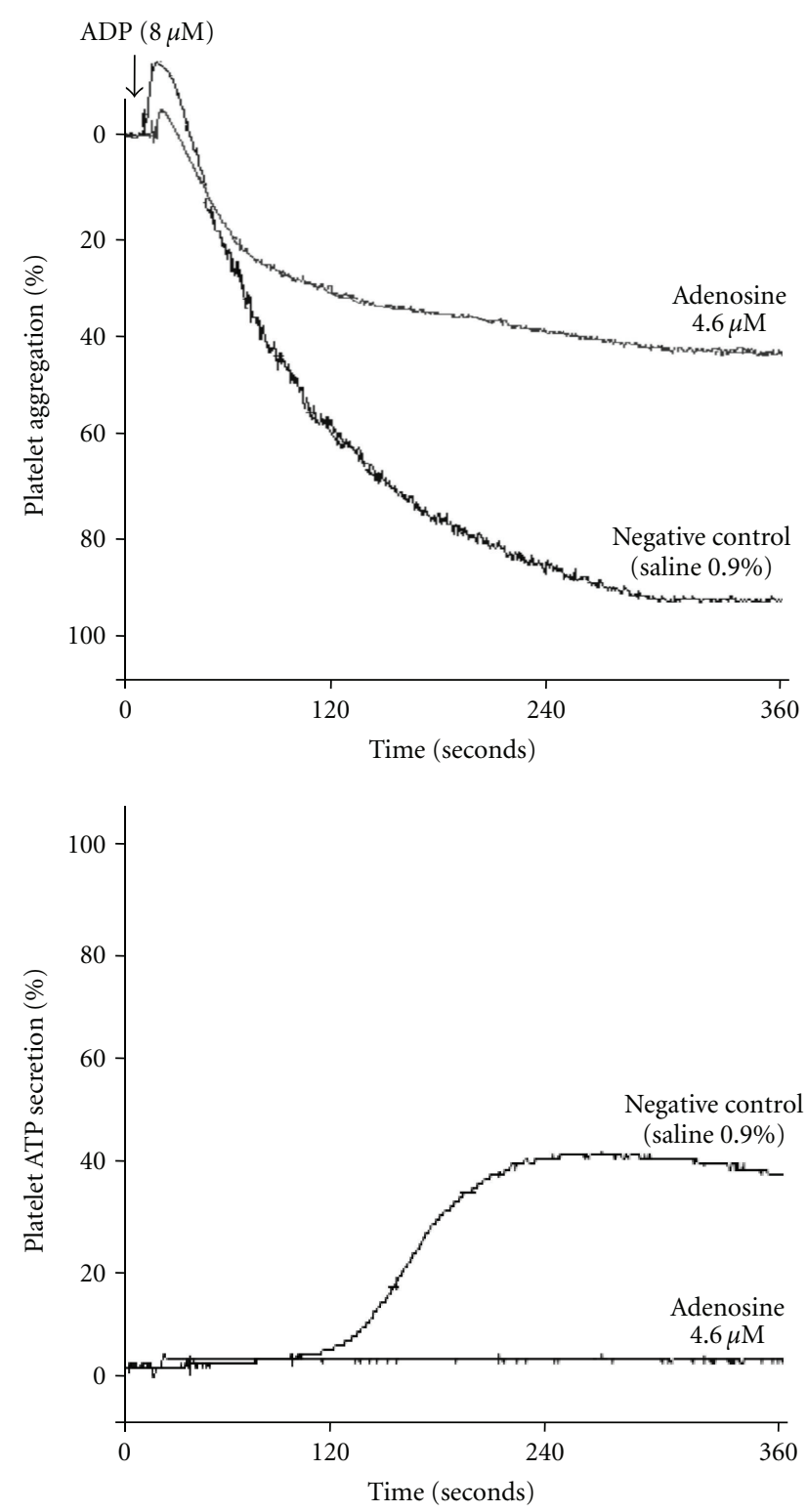

Figure 2: Adenosine $4.6 \mu \mathrm{M}$ inhibited platelet aggregation and secretion. Luciferin/luciferase reagent and then ADP $8 \mu \mathrm{M}$ were added to platelets to induce aggregation and secretion, which were recorded in real time using the lumiaggregometer.

inhibiting platelet aggregation, adenosine $457 \mu \mathrm{M}$ displayed a net lag time of $53 \pm 2 \mathrm{~s}(P<0.05)$.

3.6. Aqueous Extract and Adenosine Inhibit Collagen-Induced Platelet Thrombus Formation under Flow Conditions. The effects of aqueous extract and adenosine on human collageninduced platelet aggregation and thrombus formation under arterial flow conditions are shown in Figure 3. After perfusion of citrate-anticoagulated blood over plaque-coated surfaces with collagen at $37^{\circ} \mathrm{C}$ with a wall shear rate of $1000 \mathrm{~s}^{-1}$ for $10 \mathrm{~min}$, rapid platelet adhesion and aggregate formation were observed (additional file Movie C1; Figure 3).

Aqueous extract and adenosine reduced collageninduced platelet adhesion and aggregate formation under controlled flow. After aqueous extract incubation of blood, the platelet coverage was inhibited by $51.6 \pm 16 \%(P<$ 0.05) (additional file Movie P1; Figure 3). For adenosine, the inhibition of platelet adhesion and aggregate formation under controlled flow were concentration dependent (data not shown), in which a concentration of $114 \mu \mathrm{M}$ was inhibited by $94.5 \pm 10 \%(P<0.05$, compared with the negative control) the platelet coverage (additional file Movie A1; Figure 3).

3.7. Platelet Antiaggregating Activity in Ripe Tomato Fruits and Its by-Products of Processing. The inhibition of platelet aggregation ADP-induced by the methanol extract of tomato skins $(1 \mathrm{mg} / \mathrm{mL})$ was $40 \pm 1 \%$ as compared to control $(P<0.05)$. Finally, the aqueous extracts $(1 \mathrm{mg} / \mathrm{mL})$ of tomato paste and pomace inhibited platelet aggregation ADP 


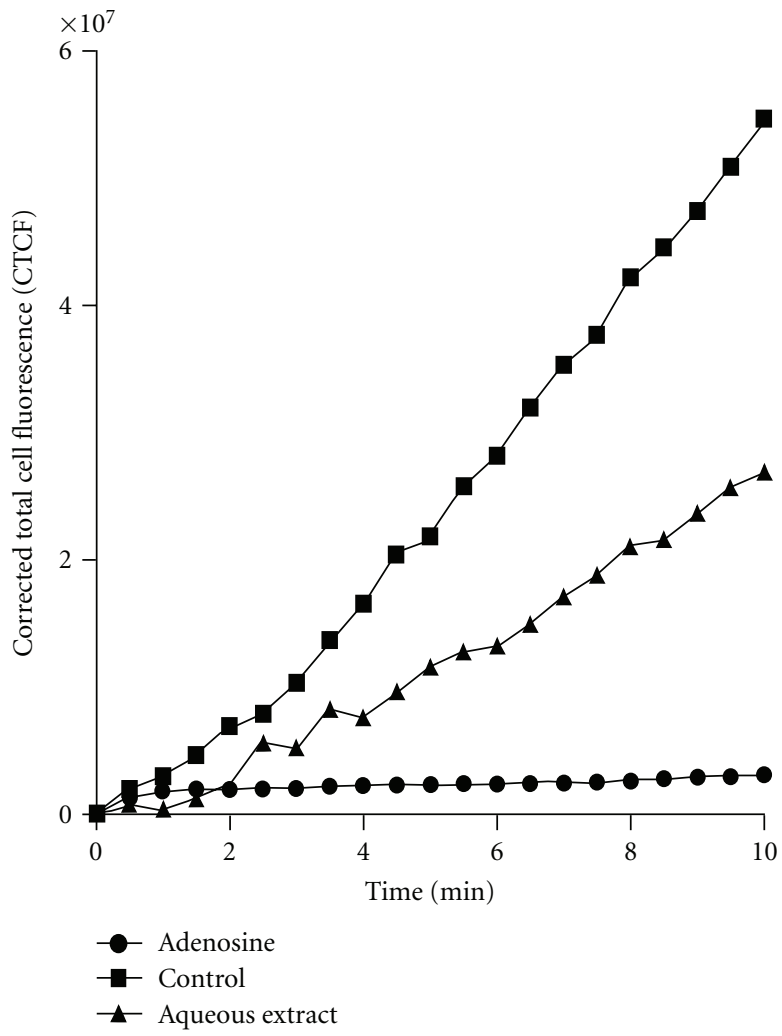

(a)

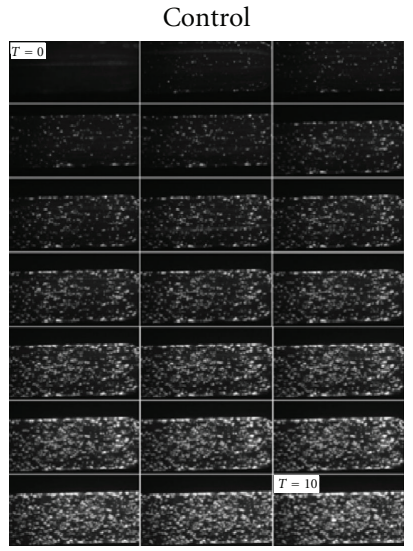

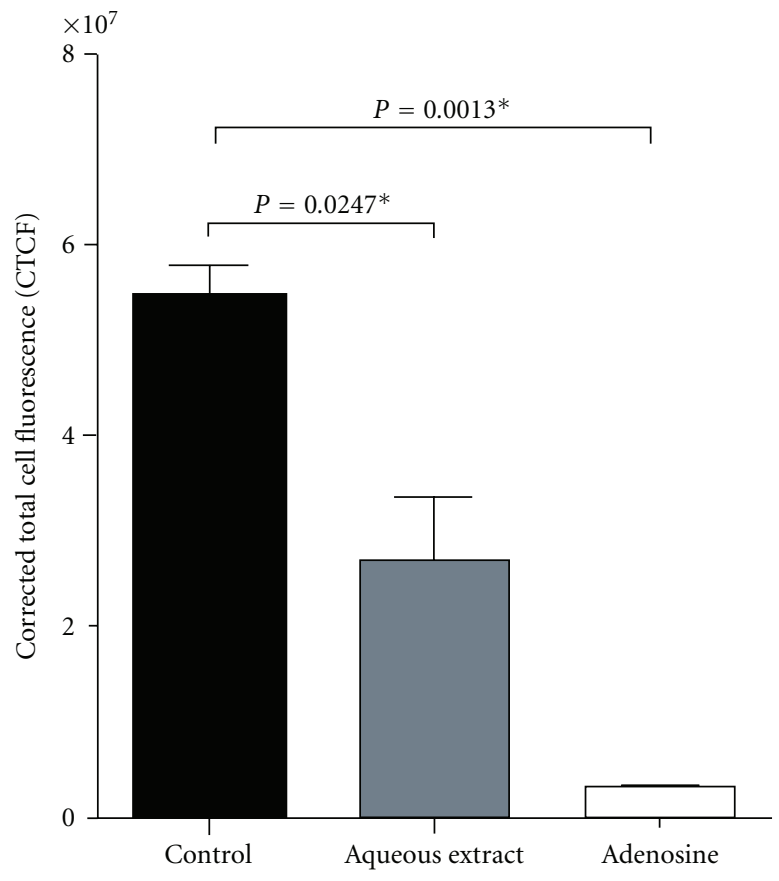

(b)
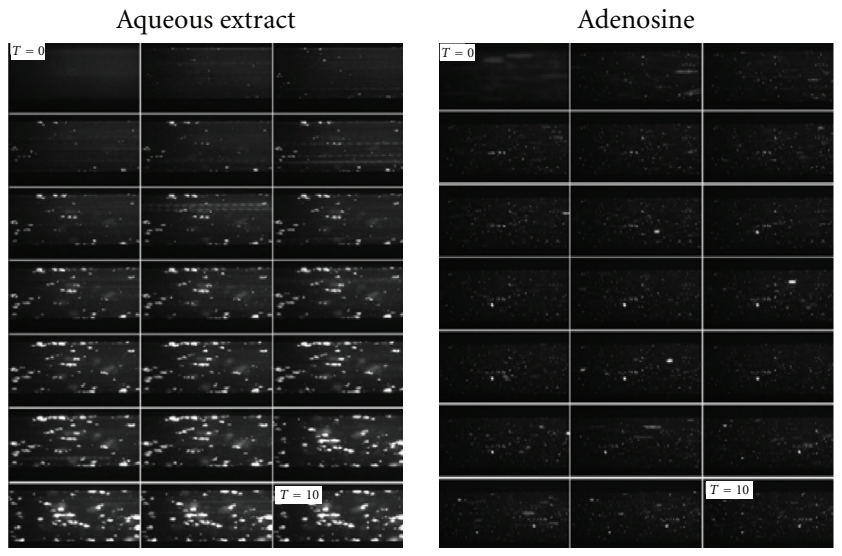

(c)

FIGURE 3: Effect of aqueous extract and adenosine on collagen-induced platelet thrombus formation under arterial flow conditions. Citrateanticoagulated blood was preincubated with aqueous extract $(1 \mathrm{mg} / \mathrm{mL})$, adenosine $(114 \mu \mathrm{M})$, or negative control (saline $0.9 \%)$ for 1 hour and then was perfused over plaque-coated surfaces for $10 \mathrm{~min}$ at room temperature at a shear rate of $1000 \mathrm{~s}^{-1}$. (a) It shows the intensity (CTCF) over a time lapse, (b) bar diagram (values are mean $\pm \mathrm{SD}$; $n=3$ ), and (c) time lapse of $10 \mathrm{~min}$ at $1000 \mathrm{~s}^{-1}$, at $30 \mathrm{sec}$ intervals. ${ }^{*} P<0.05$.

induced in $40 \pm 3 \%$ and $35 \pm 1 \%$, respectively, as compared to negative control $(P<0.05)$ (Figure 4$)$.

3.8. Adenosine Content in Ripe Tomato Fruits and Its Processing by-Products. The adenosine content in ripe tomato fruits and its processing by-products are presented in Figure 5. Such results were calculated from an adenosine standard linear regression with a correlation coefficient of $r=0.994$. The pulp extracts (total and aqueous extracts) showed the highest content of adenosine, while tomato pomace showed the lowest amount. Finally, adenosine content in each extract was positively correlated with the inhibition of platelet function measured by platelet antiaggregant activity $(r=$ $0.94, P<0.05)$ (Figure 5).

\section{Discussion}

Epidemiological studies have provided evidence of a protective role of healthy diets in the prevention of cardiovascular disease and cancer [28]. Among the protective activities 


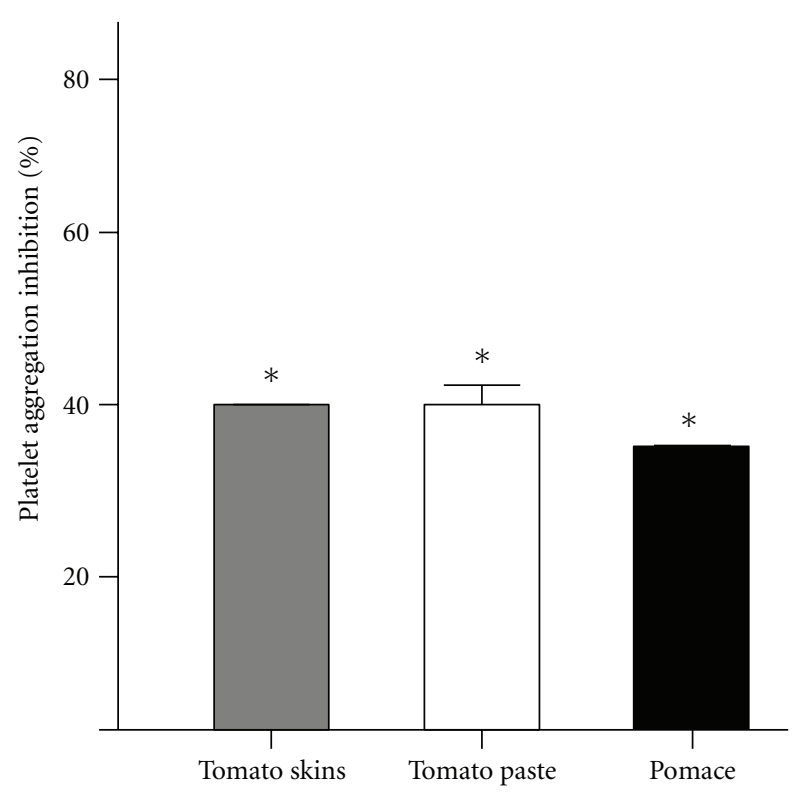

FIGURE 4: Effect of ripe tomato fruits and their by-products of processing on platelet aggregation. ADP $8 \mu \mathrm{M}$. Extracts at $1 \mathrm{mg} / \mathrm{mL}$. ${ }^{*} P<0.05$ versus negative control (saline $0.9 \%$ ).

reported for tomato, its antiplatelet activity has been associated with a decrease in the prevalence of CVD [29].

It was observed that tomatoes exert in vitro [30] and in vivo [31] antiplatelet activity through the inhibition of platelet aggregation induced by ADP and collagen, as confirmed by our research group [29]. Recently, aqueous and methanol total extracts of red tomato were found to be thermally stable in the temperature range of 20 to $100^{\circ} \mathrm{C}$ and both acid and alkali did not affect inhibition of platelet aggregation induced by ADP. The presence of lycopene in the fraction extracted with water and methanol, respectively, were extracted showing the highest antiplatelet activity [32].

For extraction and fractioning stages, were fully ripe tomato fruits used showing an optimal industrial quality based on the firmness and soluble solid content [33]. The liquid-liquid separation allowed aqueous extract to have a higher yield than ethyl acetate and petroleum ether extracts. This may occur due to the high moisture content shown in tomato pulp (28).

Thus, in this study, liquid-liquid separation allowed concentrating antiplatelet activity in the aqueous extract, discarding the presence of compound in ethyl acetate (e.g., carotenoids) and petroleum ether extracts (e.g., triterpenoids and fatty acids) [26]. Such absence of these compounds is related to the slight antioxidant activity shown by the aqueous extract, which possibly allows us to establish that in such extracts a correlation between antioxidant and antiplatelet activities does not exist [19]. Conventional chromatography techniques (Sephadex LH-20, TLC preparative, and HPLC) used in the bioassay-guided allowed obtaining extract, fraction and bioactive compound from tomatoes with antiplatelet activity.

In the present study, besides the known platelet antiaggregant activity, it was demonstrated that aqueous extract inhibits platelet function completely: platelet adhesion, secretion, and aggregation.

Adenosine at a low concentration showed a potent antiplatelet activity through the inhibition of platelet aggregation and secretion; it also displayed a net lag time in the platelet aggregation assay induced by arachidonic acid, reaching the maximum aggregation rate of $>80 \%$ at $360 \mathrm{~s}$ (data not shown). Moreover it presented inhibition of platelet thrombus formation under flow conditions. Adenosine, which can reach circulating blood from the heart, endothelium, and other tissues, is an endogenous antiaggregating substance that influences the function of circulating platelets by increasing cAMP and cGMP levels [34].

The quantitative HPLC analysis revealed that significant amounts of adenosine were contained in ripe tomato fruits and its processing by-products. Then liquid-liquid separation, the major content of adenosine in aqueous extract, is due to the affinity characteristics of this compound by polar extracts. On the other hand, the lowest adenosine content in tomato paste (rich in tomato pulp) may be due to the heat treatment, and to a lesser extent, because the sterilization stage during the paste-making process affects this compound negatively [35]. Tomato pomace is obtained as a by-product from processing tomatoes into fluid and pasty products such as tomato juice, sauce, and paste. Its content of adenosine is due to that it contains about $44 \%$ seeds and $56 \%$ of peels [36].

The present study confirms the antiplatelet activity of tomatoes; thus, based on the moisture rate and quantitative analysis of HPLC, $1 \mathrm{~kg}$ of fresh tomato contains about 30times more adenosine than lycopene. So, tomato fruits and their by-products of processing have other bioactive compound, biologically complementing the activities of lycopene [37].

Finally, according to a correlation study, knowing the adenosine content in different extracts is possible to establish the degree of platelet aggregation inhibition. From these results, extracts/fractions of ripe tomato fruits and their by-products of processing may be referred to as functional food and functional ingredients containing a compound that inhibits platelet function with a potent preventive effect on thrombus formation, as those that occur in stroke.

\section{Conclusion}

Through bioassay guided on the basis of antiplatelet activity, was possible to isolate, identify, and determine a bioactive compound with a complementary biological activity to that of the lycopene. The antiplatelet activity is specific for adenosine, so that, according to its content, it is possible to establish the degree of such activity in the different types of extracts. Based on the present results, extracts of ripe tomato fruits and their processing by-products due to their adenosine content may be used as functional ingredients adding antiplatelet activities to processed foods which may be supportive in the primary prevention of CVD. 


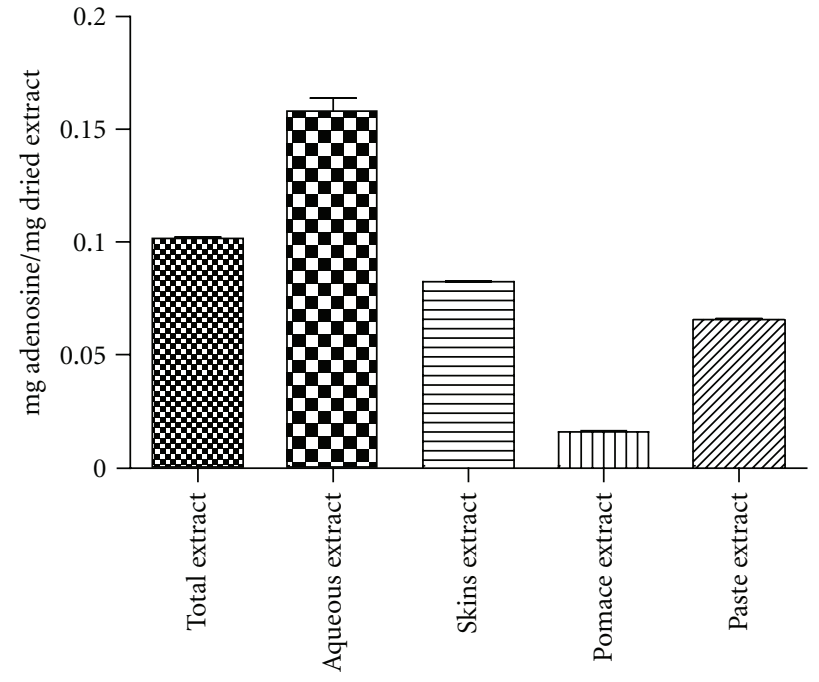

(a)

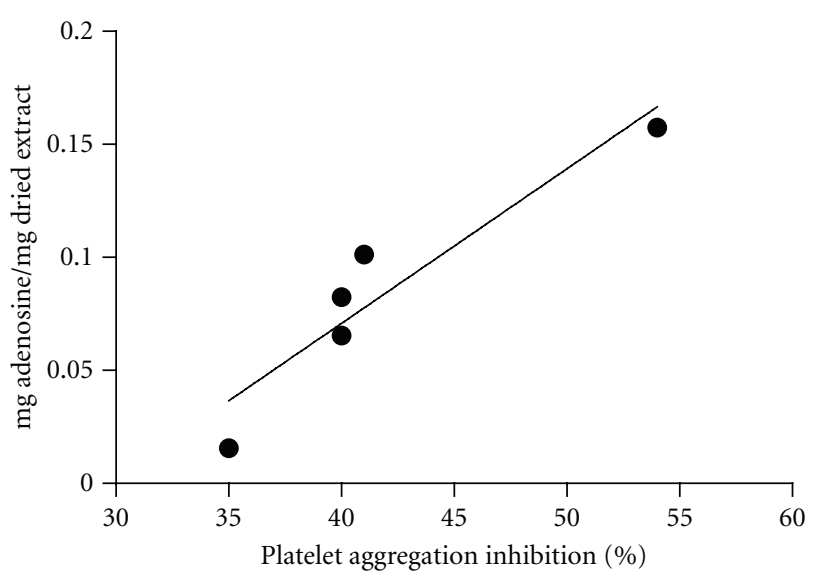

(b)

Figure 5: Quantitative analysis by HPLC of adenosine in ripe tomato fruits and their processing by-products. (a) Adenosine content expressed in mg adenosine/mg dried extract and (b) correlation coefficient and related significance between adenosine content and platelet inhibition $(r=0.94, P<0.05)$. Total and aqueous extracts are obtained from tomato pulp. All the analyses were repeated at least twice starting from independent dried extracts.

\section{Conflict of Interests}

The authors report no declarations of interest.

\section{Acknowledgment}

This work was funded by the Conicyt Regional/Gore Maule/CEAP/R09I2001.

\section{References}

[1] J. Mackay and G. Mensah, The Atlas of Heart Disease and Stroke, World Health Organization, Geneva, Switzerland, 2004.

[2] C. F. Jackson and N. K. Wenger, "Cardiovascular disease in the elderly,” Revista Española de Cardiología, vol. 64, pp. 697-712, 2011.

[3] K. S. Reddy and S. Yusuf, "Emerging epidemic of cardiovascular disease in developing countries," Circulation, vol. 97, no. 6, pp. 596-601, 1998.

[4] M. E. Marenberg, N. Risch, L. F. Berkman, B. Floderus, and U. De Faire, "Genetic susceptibility to death from coronary heart disease in a study of twins," The New England Journal of Medicine, vol. 330, no. 15, pp. 1041-1046, 1994.

[5] I. Palomo, C. Toro, and M. Alarcón, "The role of platelets in the pathophysiology of atherosclerosis (Review)," Molecular Medicine Reports, vol. 1, no. 2, pp. 179-184, 2008.

[6] K. Nishijima, J. Kiryu, A. Tsujikawa et al., "Platelets adhering to the vascular wall mediate postischemic leukocyteendothelial cell interactions in retinal microcirculation," Investigative Ophthalmology and Visual Science, vol. 45, no. 3, pp. 977-984, 2004.

[7] M. Ishikawa, D. Cooper, T. V. Arumugam, J. H. Zhang, A. Nanda, and D. N. Granger, "Platelet-leukocyte-endothelial cell interactions after middle cerebral artery occlusion and reperfusion," Journal of Cerebral Blood Flow and Metabolism, vol. 24, no. 8, pp. 907-915, 2004.

[8] S. M. Schoenwaelder, Y. Yuan, E. C. Josefsson et al., "Two distinct pathways regulate platelet phosphatidylserine exposure and procoagulant function," Blood, vol. 114, no. 3, pp. 663666, 2009.

[9] J. E. Italiano Jr., J. L. Richardson, S. Patel-Hett et al., "Angiogenesis is regulated by a novel mechanism: pro- and antiangiogenic proteins are organized into separate platelet $\alpha$ granules and differentially released," Blood, vol. 111, no. 3, pp. 1227-1233, 2008.

[10] E. Fuentes, F. Fuentes, V. Andrés et al., "Role of platelets as mediators that link inflammation and thrombosis in atherosclerosis," Platelets. In press.

[11] Z. M. Ruggeri, "Mechanisms initiating platelet thrombus formation," Thrombosis and Haemostasis, vol. 78, no. 1, pp. 611-616, 1997.

[12] R. Estruch, M. A. Martínez-González, D. Corella et al., "Effects of a Mediterranean-style diet on cardiovascular risk factors a randomized trial," Annals of Internal Medicine, vol. 145, no. 1, pp. 1-11, 2006.

[13] T. A. Pearson, S. N. Blair, S. R. Daniels et al., "AHA guidelines for primary prevention of cardiovascular disease and stroke: 2002 update: consensus panel guide to comprehensive risk reduction for adult patients without coronary or other atherosclerotic vascular diseases," Circulation, vol. 106, no. 3, pp. 388-391, 2002.

[14] I. Palomo, E. Leiva, and M. Vásquez, Dieta Mediterranea: Prevención de las Enfermedades Cardiovasculares, Universidad de Talca Press, Talca, Chile, 2007.

[15] V. Koleckar, E. Brojerova, Z. Rehakova et al., "In vitro antiplatelet activity of flavonoids from Leuzea carthamoides," Drug and Chemical Toxicology, vol. 31, no. 1, pp. 27-35, 2008.

[16] A. N. Khan, I. Fatima, U. A. Khaliq et al., "Potent anti-platelet constituents from centaurea iberica," Molecules, vol. 16, no. 3, pp. 2053-2064, 2011. 
[17] I. Palomo, E. Fuentes, T. Padró, and L. Badimon, "Platelets and atherogenesis: platelet antiaggregating activity and endothelial protection from tomatoes (Solanum lycopersicum L.)," Experimental and Therapeutic Medicine, vol. 3, pp. 577-584, 2012.

[18] S. M. Renaud and J. T. Luong-Van, "Seasonal variation in the chemical composition of tropical Australian marine macroalgae," Journal of Applied Phycology, vol. 18, no. 3-5, pp. 381-387, 2006.

[19] E. Fuentes, L. Astudillo, L. Guzmán et al., "Antioxidant and antiplatelet activities in extracts from green and fully ripe tomato fruits (Solanum lycopersicum L.) and pomace from industrial tomato processing," Blood Coagulation \& Fibrinolysis. In press.

[20] X. F. Xue, J. H. Zhou, L. M. Wu, L. H. Fu, and J. Zhao, "HPLC determination of adenosine in royal jelly," Food Chemistry, vol. 115, no. 2, pp. 715-719, 2009.

[21] P. Molyneux, "The use of the stable free radical diphenylpicrylhydrazyl (DPPH) for estimating antioxidant activity," Songklanakarin Journal of Science and Technology, vol. 26, pp. 211219, 2004.

[22] G. V. Born and M. J. Cross, "The aggregation of blood platelets," The Journal of Physiology, vol. 168, pp. 178-195, 1963.

[23] Z. Li, G. Zhang, G. C. Le Breton, X. Gao, A. B. Malik, and X. $\mathrm{Du}$, "Two waves of platelet secretion induced by thromboxane A2 receptor and a critical role for phosphoinositide 3-kinases," Journal of Biological Chemistry, vol. 278, no. 33, pp. 3072530731, 2003.

[24] C. G. Conant, M. A. Schwartz, T. Nevill, and C. IonescuZanetti, "Platelet adhesion and aggregation under flow using microfluidic flow cells," Journal of Visualized Experiments, no. 32, article 1644, 2009.

[25] Y. Dumas, M. Dadomo, G. Di Lucca, and P. Grolier, "Effects of environmental factors and agricultural techniques on antioxidant content of tomatoes," Journal of the Science of Food and Agriculture, vol. 83, no. 5, pp. 369-382, 2003.

[26] M. Gómez-Romero, A. Segura-Carretero, and A. FernándezGutiérrez, "Metabolite profiling and quantification of phenolic compounds in methanol extracts of tomato fruit," Phytochemistry, vol. 71, no. 16, pp. 1848-1864, 2010.

[27] C. Demicheli, L. S. Santos, C. S. Ferreira et al., "Synthesis and characterization of $\mathrm{Sb}(\mathrm{V})$-adenosine and $\mathrm{Sb}(\mathrm{V})$-guanosine complexes in aqueous solution," Inorganica Chimica Acta, vol. 359, no. 1, pp. 159-167, 2006.

[28] P. M. Kris-Etherton, K. D. Hecker, A. Bonanome et al., "Bioactive compounds in foods: their role in the prevention of cardiovascular disease and cancer," American Journal of Medicine, vol. 113, supplement 9, pp. 71S-88S, 2002.

[29] C. Torres-Urrutia, L. Guzmán, G. Schmeda-Hirschmann et al., "Antiplatelet, anticoagulant, and fibrinolytic activity in vitro of extracts from selected fruits and vegetables," Blood Coagulation and Fibrinolysis, vol. 22, no. 3, pp. 197-205, 2011.

[30] A. K. Dutta-Roy, L. Crosbie, and M. J. Gordon, "Effects of tomato extract on human platelet aggregation in vitro," Platelets, vol. 12, no. 4, pp. 218-227, 2001.

[31] J. Yamamoto, T. Taka, K. Yamada et al., “Tomatoes have natural anti-thrombotic effects," British Journal of Nutrition, vol. 90, no. 6, pp. 1031-1038, 2003.

[32] E. Fuentes, L. A. Astudillo, M. I. Gutierrez et al., "Fractions of aqueous and methanolic extracts from tomato (Solanum lycopersicum L.) present platelet antiaggregant activity," Blood Coagul Fibrinolysis, vol. 23, pp. 109-117, 2012.
[33] D. M. Barrett, C. Weakley, J. V. Diaz, and M. Watnik, "Qualitative and nutritional differences in processing tomatoes grown under commercial organic and conventional production systems," Journal of Food Science, vol. 72, no. 9, pp. C441-C451, 2007.

[34] G. Anfossi, I. Russo, P. Massucco et al., "Adenosine increases human platelet levels of cGMP through nitric oxide-possible role in its antiaggregating effect," Thrombosis Research, vol. 105, no. 1, pp. 71-78, 2002.

[35] A. Vallverdú-Queralt, A. Medina-Remón, I. Casals-Ribes et al., "Effect of tomato industrial processing on phenolic profile and hydrophilic antioxidant capacity," LWT_Food Science and Technology, vol. 47, pp. 154-160, 2012.

[36] A. Schieber, F. C. Stintzing, and R. Carle, "By-products of plant food processing as a source of functional compounds-recent developments," Trends in Food Science and Technology, vol. 12, no. 11, pp. 401-413, 2001.

[37] G. R. Takeoka, L. Dao, S. Flessa et al., "Processing effects on lycopene content and antioxidant activity of tomatoes," Journal of Agricultural and Food Chemistry, vol. 49, no. 8, pp. 3713-3717, 2001. 


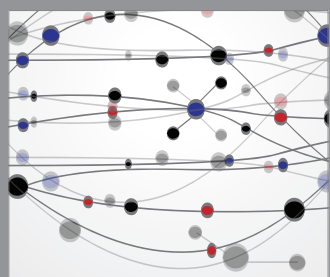

The Scientific World Journal
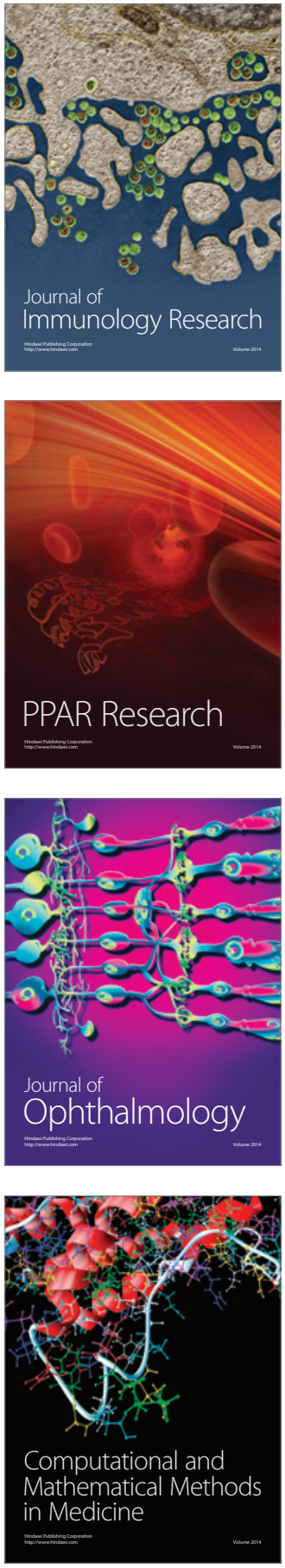

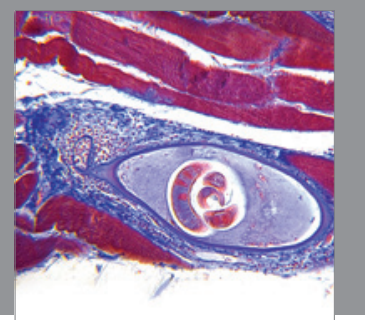

Gastroenterology

Research and Practice
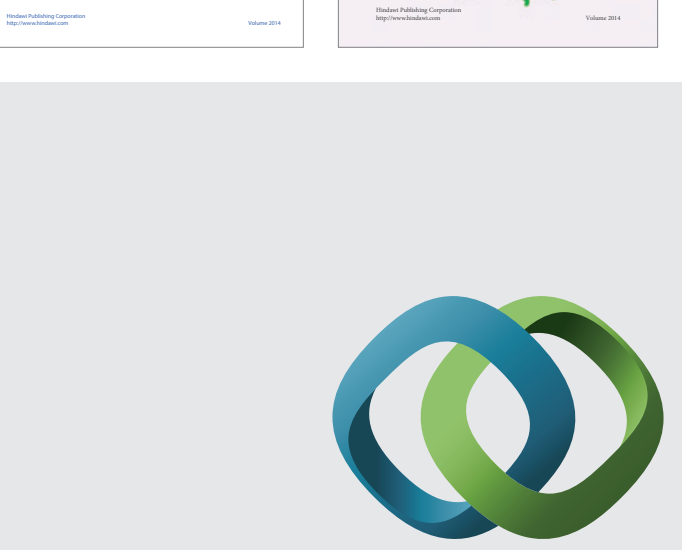

\section{Hindawi}

Submit your manuscripts at

http://www.hindawi.com
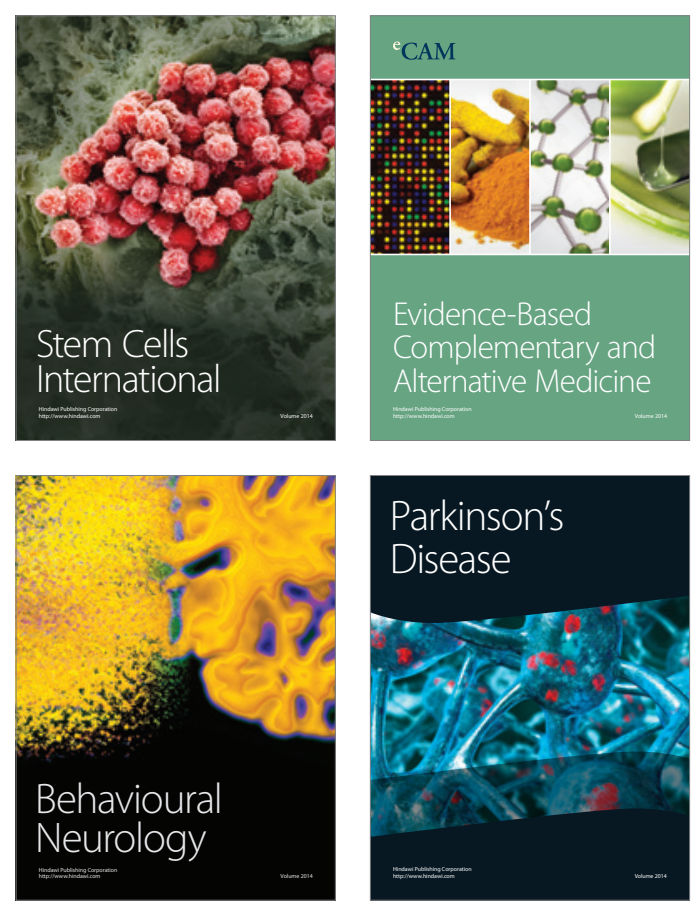

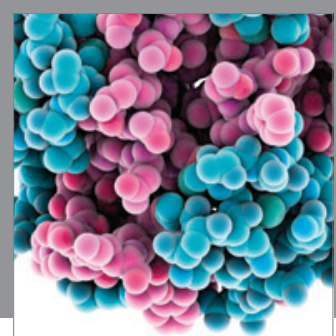

Journal of
Diabetes Research

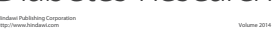

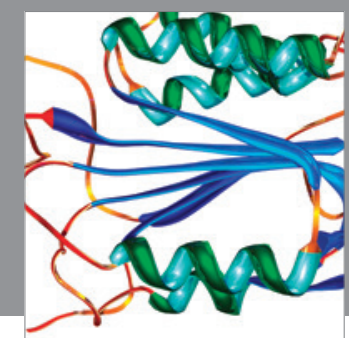

Disease Markers
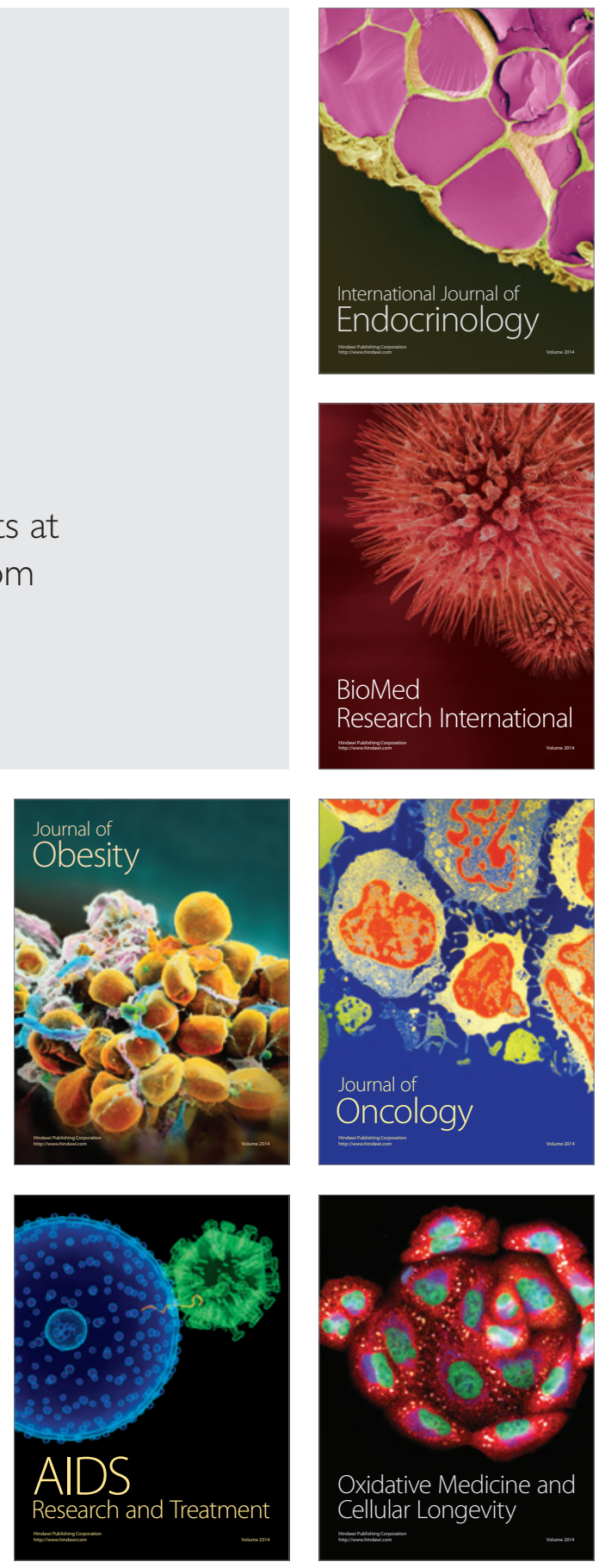